\title{
STOCHASTIC PERTURBATIONS OF CONVEX BILLIARDS
}

\author{
R. MARKARIAN, L.T. ROLLA, V. SIDORAVICIUS, F.A. TAL, M.E. VARES \\ IMERL, Facultad de Ingeniería, Universidad de la República; Universidad de Buenos Aires; \\ IMPA, Rio de Janeiro; Universidade de São Paulo; Universidade Federal do Rio de Janeiro.
}

\begin{abstract}
We consider a strictly convex billiard table with $C^{2}$ boundary, with the dynamics subjected to random perturbations. Each time the billiard ball hits the boundary its reflection angle has a random perturbation. The perturbation distribution corresponds to the physical situation where either the scale of the surface irregularities is smaller than but comparable to the diameter of the reflected object, or the billiard ball is not perfectly rigid. We prove that for a large class of such perturbations the resulting Markov chain is uniformly ergodic, although this is not true in general.
\end{abstract}

Keywords: billiard systems, random perturbations, uniform ergodicity, invariant measure

\section{INTRODUCTION}

Billiards with a stochastic perturbation of the outgoing angle are very natural models motivated by microscopic kinetic problems, theoretical computer science, etc., and have been receiving increased attention from deterministic and stochastic dynamics communities in the last decade. In most of the studied cases, the outgoing angle is either uniformly distributed, or it is chosen according to the Knudsen cosine law, see for instance [Eva01, FZ12, KY13]. These types of reflection laws are physically relevant for billiards where the micro-structure and irregularities of the boundaries have a typical length-scale larger than the diameter of the billiard ball.

In the present work we focus on the case of stochastic perturbations of the classical deterministic billiard corresponding to the physical situation where either the billiard ball is not perfectly rigid, or the scale of the surface irregularities is smaller than but comparable to the diameter of the reflected object, see Figure 1 .

Deterministic billiards on sufficiently smooth strictly convex tables are nonergodic [Laz73, KH95]. We show that, in contrast to the deterministic situation, for a certain class of physically relevant stochastic perturbations of a reflection law, the associated Markov process is uniformly ergodic, and that any probability measure converges exponentially fast to a unique invariant probability measure. We note that this is not true in general: there are examples of stochastic perturbations under which the resulting system is not ergodic. Our result holds for billiard tables which are strictly convex, with $C^{2}$ boundary, including the possibility of isolated points of null curvature. 

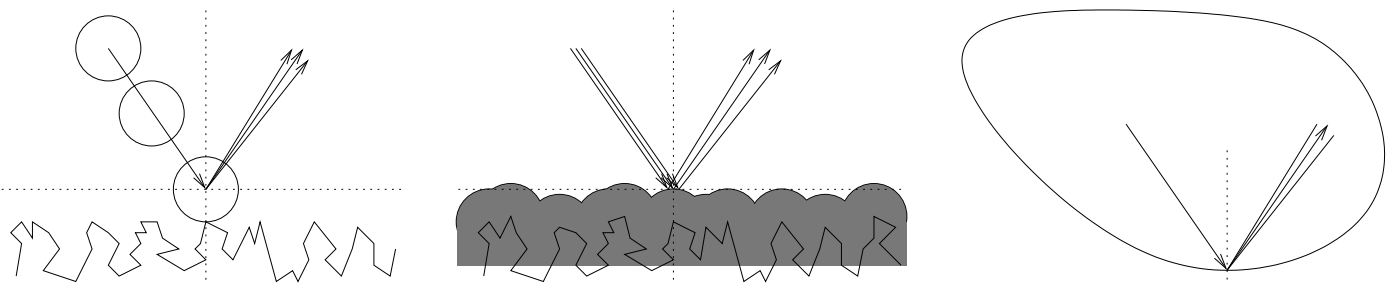

FiguRE 1. Perturbation on the reflexion angle motivated by microscopic roughness and ball radius. From left to right: microscopic paradigm of round particle reflecting on a rough surface; equivalent microscopic model of point particle reflecting on a smooth surface; effective macroscopic model.

The mathematical setup is informally described as follows. Given a prescribed family of independent random variables $\left\{Y_{\theta}\right\}_{\theta \in[0, \pi]}$, the dynamics obeys a stochastic rule. If the outgoing angle after a deterministic collision would have been $\theta$, it is taken as $\theta+Y_{\theta}$ instead. The family $\left\{Y_{\theta}\right\}_{\theta \in[0, \pi]}$ is chosen in such a way that typically the influence of $Y_{\theta}$ is negligible compared to $\theta$. However, it becomes substantial when the incidence angle gets too small. The latter property reflects an increased sensitivity to surface rugosity.

Stochastic perturbations of classical billiard systems have been proposed and studied before, see [CPSV09, FZ10, CF12, CCF13, Yar13. Yet, we are not aware of prior studies of perturbations similar to those considered here.

This paper is divided as follows. In Section 2 we describe the model and state the main result. Basic properties of convex billiard tables are briefly reviewed in Section 3. The main technical result of this paper is Proposition 5, which is stated in Section 4, and from which Theorem 1 is derived at end of this section. Section 5 is dedicated to the main technical proofs, culminating with the proof of Proposition 5 ,

\section{MODELS AND RESUlT}

We begin with the description of the deterministic billiard in $D$, a connected domain in $\mathbb{R}^{2}$. We assume throughout this paper that $D$ is strictly convex with $C^{2}$ boundary. Notice that isolated points with null curvature are allowed.

The billiard in $D$ is the dynamical system describing the free motion of a point mass inside $D$ with elastic reflections at its boundary $\Gamma$. Let $n(q)$ be the unit normal to the curve $\Gamma$ at the point $q$ pointing towards the interior of $D$. The phase space of such a dynamical system is $\{(q, v): q \in \Gamma,|v|=1,\langle v, n(q)\rangle \geqslant 0\}$.

The image of a point $\left(q_{0}, v_{0}\right)$ by the deterministic billiard map $T$ is denoted by

$$
T\left(q_{0}, v_{0}\right)=\left(q_{1}, v_{1}\right)
$$


and defined as follows. First, $q_{1}$ is the point where the oriented line through $\left(q_{0}, v_{0}\right)$ hits $\Gamma$. Finally, $v_{1}$ is the velocity vector after the reflection at $q_{1}$, i.e. $v_{1}=v_{0}-$ $2\left\langle n\left(q_{1}\right), v_{0}\right\rangle n\left(q_{1}\right)$.

We take the set of coordinates $(s, \theta)$, where $s$ is the arc-length parameter along $\Gamma$ and $\theta \in[0, \pi]$ is the angle between $v$ and the oriented tangent line to the boundary at $q$. The phase space under these coordinates is given by the cylinder

$$
M=\{(s, \theta): 0 \leqslant s<|\Gamma|, 0 \leqslant \theta \leqslant \pi\} .
$$

For $x=(s, \theta) \in M$, we write $s(x)=s, \theta(x)=\theta$, and also $q(x)$ for the corresponding point in $\Gamma$. The map $T$ is a diffeomorphism defined on the compact set $M$ with fixed points at $\partial M=\{(s, \theta): \theta=0$ or $\pi\}$.

Moreover, $T$ is a twist diffeomorphism. This means that the image of any vertical line $(s=$ constant $)$ is a smooth curve with slope positive and bounded away from infinity, see [KH95, Section 9.2].

If $D$ is strictly convex with sufficiently smooth boundary, by KAM theory there exist invariant curves of the billiard map as close as we want to the boundary $\partial M$, see the end of Section 3. Therefore, if the initial angle is small it remains small along the whole trajectory. We show that this regularity can be broken using arbitrarily small random perturbations.

We consider the system with random perturbations that act on the outgoing angle, independently of the position, by adding a random variable to $\theta$. Our choices of perturbations are motivated by physical situations where either the scale of the surface irregularities is smaller than but comparable to the diameter of the reflected object, or the billiard ball is not perfectly rigid. Fix $0<\epsilon<\frac{\pi}{2}$. For $\theta$ away from 0 and $\pi$, we take the probability density of the outgoing angle as a constant on the interval $[\theta-\epsilon, \theta+\epsilon]$ (the correct law derived from Figure 1 would not have constant density, but this is irrelevant for the qualitative behavior of the model). The particular choice of perturbation becomes more delicate when the collision angle is close to the extremes. We give three specific examples to illustrate possible behaviors.

Example 1. For every point $x=(s, \theta) \in M$, define $\theta^{\epsilon}:=\min \{\max (\theta, \epsilon), \pi-\epsilon\}$, and consider the measure $Q_{x}^{\epsilon}$ on $M$ given by

$$
Q_{x}^{\epsilon}(A)=\int_{\theta^{\epsilon}-\epsilon}^{\theta^{\epsilon}+\epsilon} \mathbb{1}_{A}(s, u) \frac{1}{2 \epsilon} \mathrm{d} u
$$

In other words, the random outgoing angle is distributed uniformly on $\left[\theta^{\epsilon}-\epsilon, \theta^{\epsilon}+\epsilon\right]$.

Example 2. Outgoing angle uniformly distributed on $[\max \{\theta-\epsilon, 0\}, \min \{\theta+\epsilon, \pi\}]$

Example 3. Outgoing angle uniformly distributed on $[0,2 \theta]$ for $\theta<\epsilon$, and defined analogously for $\theta>\pi-\epsilon$. 
In Examples 1 and 2, the outgoing angle is uniformly distributed over an interval whose length is at least $\epsilon$ : for $\theta<\epsilon$, Example 1 replaces $\theta$ by a uniform on $[0,2 \epsilon]$, whereas Example 2 replaces $\theta$ by a uniform on $[0, \theta+\epsilon]$. Example 3, on the other hand, is very different in nature. For a trajectory where the outgoing angle would be almost tangent to the boundary $\Gamma$, the replacement by a uniform on $[0,2 \theta]$ keeps it very close to being tangent. We find the first two examples natural to justify for a physical situation of a rigid sphere hitting a rough surface, as shown in Figure 2 , From now on we concentrate on Example 1, bearing in mind that all the arguments translate seamlessly to Example 2 or other similar cases.

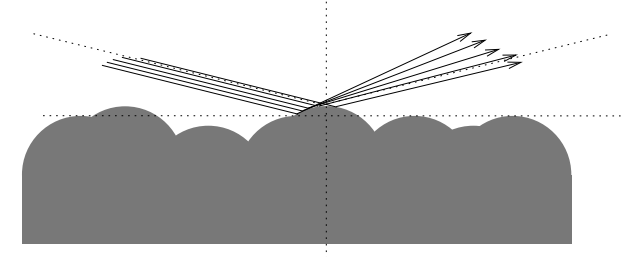

FiguRE 2. Microscopic view of a rigid ball colliding with a rough surface. Or, equivalently, point mass colliding with an irregular but microscopically smooth surface. When the incidence angle is very small, the randomness of the outgoing angle is no longer determined by how the particle hits a given protuberance: it is rather sensitive to which parts of the protuberances are visible to the particle. For the reflected angle to be yet smaller than the incidence angle, it would require the particle to hit the surface on the back side, which becomes less likely as the incidence angle tends to zero.

Denote by $\mathcal{B}$ the Borel $\sigma$-field on $M$, and $\mathcal{P}$ the set probability measures on $\mathcal{B}$, and the total variational distance on $\mathcal{P}$ denoted by $\|\mu-\nu\|=\sup _{A \in \mathcal{B}}|\mu(A)-\nu(A)|$.

Definition. The stochastic perturbation of the map $T$ is given by the transition kernel $P_{\epsilon}(x, A)=Q_{T x}^{\epsilon}(A), x \in M, A \in \mathcal{B}$.

Observe that $P_{\epsilon}(., A)$ is a measurable function for every $A \in \mathcal{B}$, and $P_{\epsilon}(x,$.$) is a$ measure on $\mathcal{B}$ for every $x \in M$.

The push-forward operator $\mu \mapsto \mu P_{\epsilon}$ for $\mu \in \mathcal{P}$ is given by

$$
\mu P_{\epsilon}(A)=\int_{M} \mu(\mathrm{d} x) P_{\epsilon}(x, A),
$$

and we say that $\mu \in \mathcal{P}$ is invariant for $P_{\epsilon}$ if $\mu P_{\epsilon}=\mu$, see Section 4 .

Theorem 1. Suppose that $D$ is strictly convex and its boundary $\Gamma$ is $C^{2}$. For each $0<\epsilon<\frac{\pi}{2}$, there exists a unique invariant measure $\nu_{\epsilon}$ for $P_{\epsilon}$, and moreover there exists $\gamma>0$ such that $\left\|\mu P_{\epsilon}^{n}-\nu_{\epsilon}\right\| \leqslant e^{-\gamma n}$ for all $\mu \in \mathcal{P}$ and $n \in \mathbb{N}$.

Theorem 1 remains valid, with essentially the same proof, for a much broader class of distributions. What is important is that the probability density of the outgoing 
angle is bounded from below on some interval around $\theta$ whose length is also bounded from below.

Yet, the validity of the Theorem 1 is far from being general. Stochastic dynamics constructed using the distribution of Example 3 is not only non-ergodic, but it gets quickly absorbed by a random point at the boundary $\partial M$. We omit the proof of this fact.

\section{BASIC PROPERTIES OF DETERMINISTIC BILLIARDS IN CONVEX TABLES}

The map $T$ preserves the probability measure $\nu$ defined by $d \nu=\frac{1}{2|\Gamma|} \sin \theta d s d \theta$. It satisfies an involution property: if $I: M \rightarrow M$ is defined by $I(q, \theta)=(q, \pi-\theta)$, then $T^{-k} \circ I=I \circ T^{k}, k \in \mathbb{Z}$.

If $k \geqslant 2, T$ is a $C^{k-1}$ diffeomorphism in the interior of $M$. For every $x \in M$, the matrix of the differential $D_{x} T$ reads as

$$
D_{x} T=\left(\begin{array}{cc}
\frac{\kappa(x) t(x)-\sin \theta(x)}{\sin \theta(T x)} & \frac{t(x)}{\sin \theta(T x)} \\
\frac{\kappa(T x) \kappa(x) t(x)-\kappa(T x) \sin \theta(x)}{\sin \theta(T x)}-\kappa(x) & \frac{\kappa(T x) t(x)}{\sin \theta(T x)}-1
\end{array}\right),
$$

where $\kappa(x)$ is the curvature of $\Gamma$ at $q(x)$, and $t(x)$ is the distance between $q(x)$ and $q(T x)$. Both values are continuous for $x \in \operatorname{int} M$, the interior of $M$ (see [CM06] for a proof, noticing that here we use a different parametrization of angles).

Lemma 2. If $T(x)=\left(s_{1}(x), \theta_{1}(x)\right)$, then $\left(\frac{\partial s_{1}}{\partial \theta}\right)^{-1}$ can be continuously extended to the boundary of $M$. In particular, $\frac{\partial s_{1}}{\partial \theta}$ is bounded away from zero.

Proof. Using the expression of $D_{x} T$ we obtain that $\frac{\partial s_{1}}{\partial \theta}=\frac{t(x)}{\sin \theta_{1}(x)}$. If $x_{n}$ is a sequence converging to a boundary point $\bar{x}$, then both $t\left(x_{n}\right)$ and $\sin \theta_{1}\left(x_{n}\right)$ converge to 0 , but in this case $\frac{\kappa\left(T x_{n}\right) t\left(x_{n}\right)}{2 \sin \theta\left(T x_{n}\right)}$ tends to 1 and the $\left(\frac{\partial s_{1}}{\partial \theta}\right)^{-1}$ goes to $\kappa(T \bar{x}) / 2$.

We remark that $T$ being a twist map holds on more general tables, in particular if $D$ is convex with $C^{1}$ boundary. In this case the map $T$ is an homeomorphism in $\operatorname{int} M$ that can be extended defining $T x=x$ for every $x \in \partial M$. For this extension $x \mapsto q(T(x))$ is not continuous in $x$ if $q(x)$ is in the interior of a segment of the boundary, but $x \mapsto \theta(T(x))$ is nonetheless continuous.

For any $0<a<\frac{\pi}{2}$, we define the cylinder $M_{a}=[0,|\Gamma|) \times[a, \pi-a]$.

Lemma 3. Suppose that $D$ is convex with $C^{1}$ boundary. Given $\epsilon>0$, there exist $0<c_{2}<c_{1}<\epsilon$ satisfying the following conditions: $M_{\epsilon} \subset T\left(M_{c_{1}}\right), M_{c_{1}} \subset T^{2}\left(M_{c_{2}}\right)$ and $T^{2}\left(M_{c_{1}}\right) \subset M_{c_{2}}$.

Proof. Although $T$ may not be continuous in $M, \theta(T x)$ is still continuous. This implies that for sufficiently small $a \geqslant 0$ there exists $\delta_{1}(a), \delta_{2}(a)>0$ such that $M_{\delta_{1}(a)} \subset T\left(M_{a}\right) \subset M_{\delta_{2}(a)}$, and that $\delta_{1}(a), \delta_{2}(a)$ converge to 0 as $a \rightarrow 0$. 
For the deterministic billiard on a sufficiently smooth table, Lazutkin [Laz73] proved the following regularity result, see also [Dou82]. If $D$ is convex with smooth boundary and curvature bounded from below then there exists a subset $M^{\prime}$ of the phase space $M$ that has positive measure and is foliated by invariant curves; the set $M^{\prime}$ accumulates on the horizontal boundaries of $M$, the map $T$ restricted to each such curve is topologically equivalent to an irrational rotation; close to the boundary $(\theta=0$ or $\pi$ in the phase space) there is a set of positive measure with regular behavior. In fact in the circle or the ellipse the whole phase space is foliated by invariant curves. Theorem 1 shows that this regularity can be broken by an arbitrarily small stochastic perturbation.

There are billiards on convex regions with no invariant curves near the boundary. These billiards have trajectories with an infinite number of bounces in finite time. as it approaches to a point of the boundary. They can be constructed either violating the condition on the curvature or the differentiability of the boundary. In [Hal77], Halpern constructed a curve that has nowhere vanishing curvature but unbounded third derivative, and proved that there are trajectories baring this pathological behavior. Mather Mat82 constructed a convex billiard with $C^{2}$ boundary violating the condition of non-null curvature, and which has trajectories coming arbitrarily close to being positively tangent to the boundary and then arbitrarily close to being negatively tangent to the boundary.

\section{MARKOV CHAINS AND THEIR DENSITIES}

Recall that the stochastic perturbation of the map $T$ is given by the transition kernel

$$
P_{\epsilon}(x, A)=Q_{T x}^{\epsilon}(A), \quad x \in M, A \in \mathcal{B} .
$$

Let $P_{\epsilon}^{n}$ denote the $n$-th power of the kernel

$$
P_{\epsilon}^{n+1}(x, A)=\int_{M} P_{\epsilon}(x, \mathrm{~d} y) P_{\epsilon}^{n}(y, A) .
$$

$P_{\epsilon}^{n}(x,$.$) is a probability measure on \mathcal{B}$ for every $x \in M$. Moreover, as operators on $\mathcal{P}$ they satisfy $\mu P_{\epsilon}^{n}=\left(\mu P_{\epsilon}^{n-1}\right) P_{\epsilon}$, and defining $P_{\epsilon}^{0}(x, A)=\mathbb{1}_{A}(x)$, the set $\left(P_{\epsilon}^{n}\right)_{n \in \mathbb{N}_{0}}$ forms a semi-group.

Proposition 4. For the stochastic billiard map there exist density functions $p_{\epsilon}^{n}(x, y)$ such that, for every $x \in M, n \geqslant 2$, and $A \in \mathcal{B}$,

$$
P_{\epsilon}^{n}(x, A)=\int_{A} p_{\epsilon}^{n}(x, y) \mathrm{d} y .
$$

Proof. If $A=[\tilde{s}, \hat{s}] \times[\tilde{\theta}, \hat{\theta}], x=(s, \theta), T x=\left(s_{1}, \theta_{1}\right), z=\left(s_{1}, \theta^{\prime}\right), T z=\left(s_{1}^{\prime}, \theta_{1}^{\prime}\right)$ then, for $z \in \hat{T} x\left(s^{\prime}=s_{1}\right)$, we have

$$
P_{\epsilon}^{2}(x, A)=\frac{1}{4 \epsilon^{2}} \int_{\left[\theta_{1}^{\epsilon}-\epsilon, \theta_{1}^{\epsilon}+\epsilon\right]} d \theta^{\prime} \int_{[-\epsilon, \epsilon]} I_{A}\left(s_{1}^{\prime}, \theta_{1}^{\prime \epsilon}+u\right) \mathrm{d} u .
$$


Changing variables $d \theta^{\prime}=\frac{\partial \theta^{\prime}}{\partial s_{1}^{\prime}} d s_{1}^{\prime}$, and using Lemma 2, we obtain the desired density. The general case is analogous.

A Markov chain is said to satisfy Döblin's condition if there exists a probability measure $\lambda, m>0$ and $\delta_{1}<1, \delta_{2}>0$ such that, whenever $\lambda(A)>\delta_{1}$, then $P_{\epsilon}^{n}(x, A)>\delta_{2}$, for all $x \in M$.

Theorem 1 is a consequence of the following result.

Proposition 5. Suppose that $D$ is strictly convex and its boundary $\Gamma$ is $C^{2}$. Then for every $0<\epsilon<\pi / 2$, there exist $b>0$ and $N>0$ such that

$$
p_{\epsilon}^{N}(x, y)>b, \quad \forall x, y \in M .
$$

We postpone its proof to the next section.

Proof of Theorem 1. Proposition 5 implies that the chain is aperiodic $\psi$-irreducible and satisfies Döblin's condition; then it is uniformly ergodic, see [MT09, Theorem 16.2.3]. The result then follows from [MT09, Theorem 16.0.2].

\section{Proof of DöBlin's CONDItion}

Before proving Proposition 5] and Theorem 1, we need a few additional technical steps, summarized in the next two propositions.

Definition. We say that a sequence $\xi=\left(\xi_{k}\right)_{k \in[0, \ldots, l]}, \xi_{k}=\left(s_{k}, \theta_{k}\right) \in M$ is an $\epsilon$-angular perturbed orbit of length $l$ if, for all $0 \leqslant k<l, s\left(T\left(\xi_{k}\right)\right)=s_{k+1}$ and $\left|\theta\left(T\left(\xi_{k}\right)\right)-\theta_{k+1}\right|<\epsilon$

Let $\mathcal{O}_{\epsilon, l}$ denote the set of $\epsilon$-angular perturbed orbits of length $l$. For $n>0$ define

$$
\widehat{T}_{\epsilon}^{n}(x):=\left\{y: \exists \xi \in \mathcal{O}_{\epsilon, n}, \text { such that } \xi_{0}=x, \xi_{n}=y\right\} .
$$

Starting at a point $x, \widehat{T}_{\epsilon}^{n}(x)$ is the set of points that may be reached in $n$ steps by following the deterministic billiard but allowing for perturbations smaller than $\epsilon$ in the reflection angle.

Proposition 6. If $0<l<n, n \geqslant 3, y \in \widehat{T}_{\epsilon}^{n}(x)$ and there exists $z$ in the interior of $M$ such that $z \in \widehat{T}_{\epsilon}^{l}(x)$ and $y \in \widehat{T}_{\epsilon}^{n-l}(z)$, then $p_{\epsilon}^{n}$ is continuous at $(x, y)$ and $p_{\epsilon}^{n}(x, y)>0$.

Proof. From the above definition we have that $\widehat{T}_{\epsilon}^{n}(x)=\bigcup_{z \in \widehat{T}_{\epsilon}^{n-1}(x)} \widehat{T}_{\epsilon}(z)$, and if $y \in \widehat{T}_{\epsilon}^{2}(x)$ and $y$ do not belong to the boundary of $M$, then $y$ belongs to the interior of the support of $p_{\epsilon}^{2}(x,$.$) and p_{\epsilon}^{2}$ is continuous at $(x, y)$.

Proposition 7. Suppose that $D$ is convex with $C^{1}$ boundary given by the union a finite number of $C^{2}$ arcs and line segments. For every $\epsilon>0$, there exists $N \in \mathbb{N}$ such that, for all $x, y \in M$, there exists $\xi \in \mathcal{O}_{\epsilon, N}$, such that $\xi_{0}=x$ and $\xi_{N}=y$. 
Proof. We split the proof in two steps. First we show that it is possible to move between points in a given small neighborhood. Finally we use this fact to cover the whole phase space.

Step 1. By definition, if $\theta_{1}=\theta(T x) \in[\epsilon, \pi-\epsilon]$, or equivalently $T x \in M_{\epsilon}$, then $\widehat{T}_{\epsilon} x=\left\{s_{1}\right\} \times\left(\theta_{1}-\epsilon, \theta_{1}+\epsilon\right)$. In any case $\widehat{T}_{\epsilon} x=\left\{s_{1}\right\} \times\left(\theta_{1}-\epsilon, \theta_{1}+\epsilon\right) \cap M$, and $\widehat{T}_{\epsilon}^{2} x$ is a distorted rectangle. If $x$ does not belong to the boundary of $M$, then $T^{2} x$ lies in the interior of $\widehat{T}_{\epsilon}^{2} x$. Now, take $c_{2}(\epsilon)>0$, fixed by the modulus of continuity of $T$ as in Lemma 3 . If $\delta<c_{2}$ is sufficiently small, then for all $x$ in $M_{c_{2}}$, both $T^{2}\left(B_{2 \delta}(x)\right)$ and $B_{2 \delta}\left(T^{2} x\right)$ are contained in $\widehat{T}_{\epsilon}^{2} x$.

Consider a set $U \subset M_{c_{1}}$ with measure $\nu(U)>0$ and with diameter smaller than $\delta$, and let $x_{1}$ be a point in $U$. As a consequence of the Poincaré Recurrence Theorem and Birkhoff-Khinchin Ergodic Theorem, there exists a point $z$ in $U$ and $n_{U} \leqslant$ $(\nu(U))^{-1}$ with $T^{n_{U}}(z)$ in $U$.

By choice of $\delta$, we have that $z_{2}=T^{2}(z) \in \widehat{T}_{\epsilon}^{2}\left(x_{1}\right)$. From this we have that $T^{n_{U}-4}\left(z_{2}\right)=T^{n_{U}-2}(z)$ belongs to $\widehat{T}_{\epsilon}^{n_{U}-2}\left(x_{1}\right)$. Note that, by the choice of $c_{2}$, since $T^{n_{U}}(z)$ belongs to $M_{c_{1}}$, then $T^{n_{U}-2} z$ belongs to $M_{c_{2}}$ and so, again by the choice of $\delta$, the ball of radius $2 \delta$ and center $T^{2} T^{n_{U}-2}(z)$ is contained $\widehat{T}_{\epsilon}^{2}\left(T^{n_{U}-2} z\right)$ and so $U \subset \widehat{T}_{\epsilon}^{2}\left(T^{n_{U}-2} z\right) \subset \widehat{T}_{\epsilon}^{n_{U}} x_{1}$. Therefore our dynamics moves any point of $U$ to any other point in $U$ by the step $n_{U} \leqslant(\nu(U))^{-1}$.

Step 2. We partition the cylinder $M_{c_{1}}$ into $k$ rectangles $R_{1}, \ldots, R_{k}$ based on a rectangular grid of size less then $\delta / 2$, and consider the collection $Q_{1}, \ldots, Q_{l}$ of rectangles of diameter less then $\delta$, made of two adjacent rectangles $R_{i}, R_{j}$.

Let $N_{0}$ be such that $N_{0}^{-1}$ is smaller that the minimum of $\nu\left(Q_{j}\right), 1 \leqslant j \leqslant l$. Then $N_{0}$ only depends on $\epsilon$, and for each $Q_{j}$, there exists $n_{Q_{j}}<N_{0}$ such that, for any two points $x, y$ in $Q_{j}, y$ belongs to $\widehat{T}_{\epsilon}^{n_{Q_{j}}}(x)$. Let $N_{1}$ be the least common multiple of $\left\{1, \ldots, N_{0}-1\right\}$. Then, repeatedly applying the same reasoning in each rectangle, any two points in the same rectangle can be joined by a random trajectory at step $N_{1}$. More precisely, $\widehat{T}_{\epsilon}^{N_{1}} x$ contains $Q_{j}$ for each $x \in Q_{j}$.

Consider two points $x_{0}, y$ in $M_{c_{1}}$. There exists a sequence of adjacent rectangles $R_{0}, R_{1}, \ldots, R_{m}, m<k$, such that $x_{0} \in R_{0}$ and $y \in R_{m}$. Choose $x_{i} \in R_{i}, 1 \leqslant i \leqslant$ $m-1$ and let $x_{m}=y$. By construction, for any $0 \leqslant i \leqslant m-1$ there exists $j_{i}$ such that both $x_{i}$ and $x_{i+1}$ belong to $Q_{j_{i}}$. Thus $x_{i+1} \in \widehat{T}_{\epsilon}^{N_{1}}\left(x_{i}\right)$. By induction, $x_{m} \in \widehat{T}_{\epsilon}^{m N_{1}}\left(x_{0}\right)$. On the other hand, as a consequence of the recurrence of $R_{m}$ by $\widehat{T}_{\epsilon}, x_{m} \in \widehat{T}_{\epsilon}^{N_{1}}\left(x_{m}\right)$ and so we have that $x_{m} \in \widehat{T}_{\epsilon}^{n N_{1}}\left(x_{0}\right)$ for any $n>m$. Since $x_{0}$ and $y$ were arbitrary and $m<k$, for any $x$ in $M_{c_{1}}, \widehat{T}_{\epsilon}^{k N_{1}}(x)$ contains $M_{c_{1}}$.

For any $x$ in $M, \widehat{T}_{\epsilon} x$ intersects $M_{\epsilon} \subset M_{c_{1}}$, and so $\widehat{T}_{\epsilon}^{k N_{1}+1} x$ contains $M_{c_{1}}$. Now observe that if $\theta(T x)$ is smaller than $\epsilon$ or greater than $\pi-\epsilon$, then $\widehat{T}_{\epsilon} x$ contains the segment $(s(T x) \times[0, \epsilon])$ or the segment $(s(T x) \times[\pi-\epsilon, \pi])$. Then, since $M_{\epsilon} \subset$ $T\left(M_{c_{1}}\right), \widehat{T}_{\epsilon}\left(M_{c_{1}}\right)=M$ we have that $\widehat{T}_{\epsilon}^{k N_{1}+2} x=M$ for any $x \in M$. 
Proof of Proposition 5. By Proposition 17, there exists $N \geqslant 2$ such that, for all $x, y \in M, y \in \widehat{T}_{\epsilon}^{N}(x)$. In particular, by Proposition $6, p_{\epsilon}^{N}$ is continuous and strictly positive in $(x, y)$. The result follows by compactness.

\section{ACKNOWLEDGEMENTS}

R.M. would like to thank Ya. G. Sinai for suggesting this problem. R.M. also would like to thank Sônia Pinto de Carvalho (UFMG, Belo Horizonte), Gianluigi Del Magno (UTP, Lisbon), Grupo de Investigación "Sistemas Dinámicos" (CSIC, UdelaR, Uruguay), and IMPA. F.T. Was partially supported by CNPq grant 304474/2011-8 and FAPESP grant 2011/16265-8. M.E.V. was partially financed by CNPq grant 304217/2011-5 and FAPERJ grant E-24/2013-132035.

\section{REFERENCES}

[CCF13] T. Chumley, S. Cook, And R. Feres, From billiards to thermodynamics, Comput. Math. Appl., 65 (2013), pp. 1596-1613.

[CF12] S. CoOK AND R. Feres, Random billiards with wall temperature and associated Markov chains, Nonlinearity, 25 (2012), pp. 2503-2541.

[CM06] N. Chernov and R. Markarian, Chaotic billiards, vol. 127 of Mathematical Surveys and Monographs, American Mathematical Society, Providence, RI, 2006.

[CPSV09] F. Comets, S. Popov, G. M. Schütz, And M. Vachkovskaia, Billiards in a general domain with random reflections, Arch. Ration. Mech. Anal., 191 (2009), pp. 497-537.

[Dou82] R. DouAdy, Applications du théorème des tores invariants, master's thesis, Univ. Paris VII, 1982. Thèse de 3ème Cycle.

[Eva01] S. N. Evans, Stochastic billiards on general tables, Ann. Appl. Probab., 11 (2001), pp. 419-437.

[FZ10] R. Feres And H.-K. Zhang, The spectrum of the billiard laplacian of a family of random billiards, J. Stat. Phys., 141 (2010), pp. 1039-1054.

[FZ12] - Spectral gap for a class of random billiards, Comm. Math. Phys., 313 (2012), pp. 479-515.

[Hal77] B. Halpern, Strange billiard tables, Trans. Amer. Math. Soc., 232 (1977), pp. 297-305.

[KH95] A. Katok And B. Hasselblatt, Introduction to the Modern Theory of Dynamical Systems, Cambridge University Press, New York, 1995.

[KY13] K. Khanin And T. Yarmola, Ergodic properties of random billiards driven by thermostats, Comm. Math. Phys., 320 (2013), pp. 121-147.

[Laz73] V. F. LAzUtKin, Existence of caustics for the billiard problem in a convex domain, Izv. Akad. Nauk SSSR Ser. Mat., 37 (1973), pp. 186-216.

[Mat82] J. N. Mather, Glancing billiards, Ergodic Theory Dynam. Systems, 2 (1982), pp. 397403.

[MT09] S. Meyn and R. L. Tweedie, Cambridge University Press, Cambridge, 2 ed., 2009.

[Yar13] T. Yarmola, Sub-exponential mixing of random billiards driven by thermostats, Nonlinearity, 26 (2013), pp. 1825-1837. 